\title{
OTIONOMICS
}

Revista de los Estudios de Economía y Empresa

Dossier «Redes sociales, economía y empresa»

ENTREVISTA

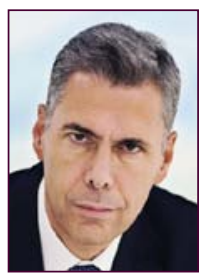

\section{Fnrique Dans: «Debemos plantear las relaciones empresariales como relaciones humanas»}

\author{
Oriol Miralbell i Joan Miquel Gomis
}

Profesores de los Estudios de Economía y Empresa (UOC)

\begin{abstract}
RESUMEN En esta entrevista, el profesor de Sistemas de Información en la IE Business School, Enrique Dans, analiza los principales cambios que las tecnologías de la información y la comunicación están provocando en las personas, organizaciones y la sociedad. El reconocido experto argumenta que las organizaciones, con la irrupción de las redes sociales, lo primero que deben plantearse es cómo desarrollar procesos de absorción de información para identificar las conversaciones de sus clientes, sus prescriptores y sus competidores en este ámbito. Explica que el desarrollo de protocolos personales en la aceptación de los cambios tecnológicos siempre va por detrás de la tecnología. Defiende que las normativas deben adaptarse a los cambios, homologando las nuevas situaciones y que hay que ayudar a las víctimas de una disrupción.
\end{abstract}

PALABRAS CLAVE redes sociales, cambio tecnológico, MOOC, competitividad

\section{We need to think of business relations in terms of human relations}

\begin{abstract}
In this interview, Enrique Dans, a Professor in Information Systems at IE Business School, looks into the main changes ICT is bringing to people, organisations, and the society. This renowned expert argues that, with the breakthrough of social media, organisations need above all to think about how to develop processes to absorb information. This will allow them to identify conversations of their customers, prescribers and competitors. Prof. Dans explains that the development of personal protocols in accepting technological change always lags behind technology. In his opinion, regulations need to adapt to change by setting the new situation as a standard; moreover, help should be provided to those suffering under disruption.
\end{abstract}

KEYWORDS social media, technological change, MOOC, competitiveness

Enrique Dans es un reconocido experto en el ámbito de la aplicación de las tecnologías de la información y la comunicación en las personas, las organizaciones y la sociedad. Profesor de Sistemas de Información en IE Business School desde el año 1990, junto a su labor docente e investigadora, se dedica también a la divulgación 
de sus análisis, ampliamente seguidos a través de su blog, y colaboraciones en medios de comunicación. Licenciado en Ciencias Biológicas por la Universidad de Santiago de Compostela, se doctoró en Sistemas de Información en UCLA y realizó estudios postdoctorales en Harvard Business School. En el 2010 publicó Todo va a cambiar, donde analiza en profundidad las transformaciones que las tecnologías están produciendo en la vida de las personas, organizaciones y en el conjunto de la sociedad.

Oriol Miralbell / Joan Miquel Gomis: Cuatro años después de la publicación del libro ¿qué balance puedes hacer?, ¿qué es lo que ha cambiado realmente?

Enrique Dans: Estamos inmersos en un proceso de cambio continuo, y en este entorno, lo que el libro venía a decir es que el desarrollo de la tecnología implica la caída de enormes barreras de entrada que estaban condicionando mucho las relaciones entre personas, las relaciones persona-empresa y las relaciones entre las empresas y sus propios empleados y los consumidores. Este nuevo entorno se basa en la bidireccionalidad, que condiciona muchas cosas: las costumbres de las personas, las relaciones empresa- consumidor... e incluso un factor tan determinante como la política, es decir, de cómo organizamos la sociedad.

O.M. /J.M.G.: En estos cuatro años, ¿la evolución de alguno de estos temas ha producido algún cambio que no habías previsto?

E.D.: Al poco tiempo de aparecer el libro se produjeron acontecimientos de carácter político que yo había considerado pero sin imaginar una evolución tan rápida. La disrupción en política ha sido sorprendente. Países que no tenían una infraestructura tecnológica ni una base amplia de abonados a redes sociales, a partir del acceso de una minoría a estas tecnologías, vivieron momentos políticos muy significativos. Ha sido muy sorprendente.

La movilidad también ha evolucionado de forma más rápida de lo previsto. Las evidencias apuntaban en este sentido, pero el movimiento ha sido más influyente de lo que imaginábamos. Ya se había previsto que el teléfono se iría convirtiendo en un ordenador de bolsillo, pero los avances han sido muy rápidos, hasta el punto de hacer posible que países con rentas medias muy bajas estén dando saltos cualitativamente significativos en los procesos de adopción de estas nuevas tecnologías móviles, en un nuevo entorno tecnológico que puede ser muy útil para su desarrollo.

El tercer elemento que me ha sorprendido se sitúa en el ámbito de la política con las revelaciones de WikiLeaks y de Edward Snowden. Me han sorprendido y preocupado a la vez la magnitud de la capacidad analítica y de espionaje que están adquiriendo las administraciones creando un escenario en el que parece que los ciudadanos necesitan protegerse de los gobiernos que ellos mismos han elegido para que les administrasen.

O.M. / J.M.G.: Las tecnologías avanzan a una velocidad que buena parte de la sociedad, la mayoría de las personas, no puede asimilar...

E.D.: El desarrollo de los protocolos personales en la aceptación de los cambios tecnológicos siempre va por detrás de la tecnología. La tecnología avanza por su cuenta, pero después su proceso va creando los protocolos de uso para que el ciudadano los asuma, entienda y adapte. Eso siempre llega después. Tardamos años en asumir que el teléfono móvil era un aparato que podíamos llevar en el bolsillo y tener desarrolladas normas de protocolo y de uso que nos permitiesen hacer un uso mínimamente razonable, que no afectara a las relaciones personales. 
O.M. / J.M.G.: Fenómenos como los de Airbnb o Uber, parecen confirmar que las normativas van más despacio incluso que estos protocolos de adopción...

E.D.: Las normativas lo que tienen que hacer es adaptarse a los cambios, homologar estas situaciones a otras parecidas y actuar con ánimo corrector. Es muy típico de las sociedades anglosajonas: se espera que haya un efecto y una vez producido se intentan corregir los posibles perjuicios que una nueva situación puede producir. En los sistemas derivados del derecho romano se plantea de otra manera y lo que se pretende es prelegislar de manera que se deduce antes de la adopción cuáles pueden ser sus efectos para prevenir y evitarlos. Y esto es imposible y absurdo porque coarta el propio desarrollo tecnológico. Entiendo que la opción del mundo anglosajón en este aspecto es mucho más razonable.

O.M. / J.M.G.: ¿Qué ocurrirá en las sociedades con esta actitud más preventiva...?

E.D.: El escenario es bastante inequívoco. Si tienes una tecnología que por el hecho de desarrollarse provoca un mejor aprovechamiento del área en la que se aplica, se acabará implantando. No puede frenarse y los intentos para obstaculizar su desarrollo serán pasajeros.

Hay que hacer entender a la víctima de una disrupción que tiene que aprender de aquel que la protagoniza. Debe mejorar su negocio a partir de ello. Así, por ejemplo, los hoteleros deben ponerse en la piel del ciudadano que elige Airbnb para reservar su alojamiento. Los directivos de las cadenas hoteleras deberían probar estos servicios y obtener sus propias conclusiones.

O.M. / J.M.G.: Víctimas de este tipo de disrupciones son los autores que claman por sus derechos de propiedad intelectual...

E.D.: El concepto de propiedad intelectual como lo teníamos entendido ya no nos sirve. Es necesario redefinirlo. Hay que darse cuenta de que los países que realmente triunfan en este contexto, otra vez con Estados Unidos a la cabeza, son los que inciden más en el desarrollo de la oferta. Se dedican a aprovechar las oportunidades que les brindan estos nuevos canales para poner más oferta al alcance de un consumidor que cada vez más dispone de más opciones para acceder a los productos culturales en formatos más variados.

Al final te das cuenta de que si distribuyes tu producto de forma racional y pensando en tu cliente, este te seguirá. Aquel que acaba descargando de forma no convencional, no era realmente tu cliente porque en ningún caso estaba dispuesto a pagar dinero por tu producto. En este caso, muchas organizaciones dan la vuelta a la situación y convierten a este cliente no convencional en un difusor y en un prescriptor del producto y utilizan las cifras de audiencia para promocionar más el producto aumentando su reputación. Se trata de diseñar modelos para que sean pocos los que no pagan, pero no intentar evitarlo hasta el extremo, porque es imposible y supone un desgaste absurdo.

O.M. / J.M.G.: En este ámbito, y en tantos otros de la sociedad de la información, ¿no serían necesarias normativas globales que superaran fronteras?

E.D.: Ciertamente hay problemáticas globales transversales que pretendemos gestionar país a país. Tenemos intereses encontrados y el hecho de que no exista un organismo regulador universal genera la mayor parte de los problemas que surgen a través de la red.

Aun así, es importante señalar que tenemos diversidad de realidades. El país que produce más productos culturales, que es Estados Unidos, tiene una realidad muy diferente a la española, cuya industria cultural vería las cosas muy diferentes si sus productos culturales fueran los más solicitados del mundo. Nada sería mejor para el 
cine o la música españoles que ser protagonista principal del hit parade de las descargas. Como no es el caso, hay quejas.

O.M. / J.M.G.: Estados Unidos podría ser un caso paradigmático de país que se adapta bien a estos procesos de cambio. ¿Qué ocurre en mercados como el español?

E.D.: Efectivamente, Estados Unidos puede ser un paradigma de país que asimila los desarrollos que plantean sus propias start up y se ha acostumbrado a procesos de adopción muy rápidos y ágiles.

En el caso de España tiene unos procesos de adopción tecnológica interesantes, a veces rapidísimos. Desde el punto de vista individual, somos muy influenciables y aparentemente tenemos pocas barreras de adopción. Pero en las organizaciones esta adopción suele ser más lenta. Especialmente en el caso del tejido empresarial que forman aquellas empresas subsidiarias de organizaciones radicadas en el extranjero. En estos casos los procesos de adopción se gestionan de manera lenta al estar condicionados por las decisiones que se toman en las sedes centrales. Esto nos acaba generando una economía muy centrada en los servicios y poco pendiente de la adopción de la tecnología.

Por otra parte, al margen de esta barrera nos enfrentamos también a tópicos. A veces hay directivos que consideran, por ejemplo, que las redes sociales son herramientas que solo sirven para perder el tiempo y que hay que prohibirlas en las organizaciones para que los empleados no las utilicen en horas de trabajo. No hacen un análisis mínimamente serio de las ventajas que las redes sociales les pueden generar. Nosotros, en los cursos de alta dirección, nos planteamos romper este tipo de tópicos.

En este sentido, las barreras son conceptuales. No se puede esperar que una empresa se adapte a estos cambios si su cúpula directiva no es capaz de ver la utilidad de las herramientas que les pueden facilitar precisamente esta adaptación. Es fundamental desarrollar procesos de innovación interna: para gestionar la información y la innovación.

\section{O.M. / J.M.G.: ¿En qué aspectos estratégicos clave las redes sociales transforman las organizaciones?}

E.D.: Lo primero que deben plantear las organizaciones es cómo desarrollar los procesos de absorción de información para identificar las conversaciones que sus clientes, sus prescriptores y sus competidores están manteniendo ahí fuera. Información que hay que saber destilar intentando evitar la clásica "parálisis por el análisis". Normalmente este tipo de decisiones se toman ante la primera crisis informacional que provoca un cliente molesto que ha expuesto en la red social su malestar con nuestra organización. Hay que establecer sistemas para valorar la gravedad de este tipo de crisis, ya que en muchos casos sus efectos son pasajeros.

A partir de aquí, hay que plantearse que además de tener información sobre lo que está ocurriendo, además de tener orejas para escuchar las conversaciones, debes tener boca. Necesitas desarrollar formas de hablar para conseguir que se te acepte como interlocutor cuando hablas de ti y de tus propios productos. De hecho, los directivos de una organización deben dejar de ser solo gestores y buenos trabajadores: deben ser también prescriptores, referentes e influenciadores. Es un nuevo escenario en el que el directivo asume un nuevo rol con las redes sociales.

Y esto vale para cualquier tipo de organización, grande o pequeña, e incluso para las start up, que no pueden esperar a tener presencia en las redes sociales cuando se lanzan al mercado. Deben estar presentes en las redes mucho antes de lanzarse al mercado.

O.M. / J.M.G.: ¿Cuándo la gestión de estos servicios debe integrarse en la empresa y cuándo puede subcontratarse?

E.D.: Cuando tu encargas a una agencia que te lleve el SEO, que desarrolle tu marca... inicialmente puede tener sentido como aprendizaje cuando la organización empieza en esto. También se puede trabajar con una agencia 
cuando se busca identificar cosas nuevas con la idea de que la agencia alimente a la organización de innovaciones respecto a herramientas, tendencias, desarrollos.

Pero cuando estamos tratando con información crítica para la organización, la agencia no puede darme el servicio que sí puede darme un departamento interno. En sectores como el turístico o el periodístico donde el SEO es estratégico, es raro ver a empresas que subcontraten su gestión. Tienen su departamento propio, porque si dejas que un tercero maneje datos sensibles como estos sobre tu empresa seguro que pierdes dinero. Creo que este tipo de agencias en muchos casos están perdiendo su sentido: deben sofisticarse con el cliente, crecer con él, y tratar de participar en la creación de ventajas competitivas.

O.M. / J.M.G.: En este escenario, ¿las grandes organizaciones, con mayor capacidad de inversión, no cuentan con una ventaja competitiva con relación a las pymes?

E.D.: En la actualidad existen herramientas analíticas muy eficientes de tipo do it yourself que pueden ser utilizadas de forma eficiente por organizaciones de pequeña dimensión. La primera fuente de información es la que te pueden proporcionar tus clientes y los datos que te facilitan se pueden ya gestionar con herramientas sencillas. Son herramientas de analítica web, web social, análisis de la competencia o de identificación de la marca. Las herramientas se alimentan de datos de las redes sociales, con formatos fáciles de usar y a costes asumibles para pymes.

El siguiente paso es situarse en el aprendizaje del denominado aprendizaje automático (machine learning). Se va también en este campo hacia un modelo de herramientas sencillas y visuales para que la máquina busque algoritmos, análisis predictivos, sin necesidad de disponer de programas complejos y muy caros que requieren además de personal muy especializado para su gestión y que solo están al alcance de las grandes organizaciones. Estas nuevas herramientas se alimentan de datos para la búsqueda de algoritmos que ayuden a la toma de decisiones en la definición, entre otros, de modelos de eficiencia o análisis predictivos.

O.M. / J.M.G.: Pero la tecnología por sí sola no garantiza el éxito...

E.D.: La red a lo que te lleva es a la universalización. Las ideas que funcionan surgen de una necesidad y a menudo no requieren de una gran tecnología. Es el caso de Uber que no requiere de una tecnología muy desarrollada con muchas horas de programación. Sin embargo, si el creador de Uber hubiera sido español, no hubiera tenido forma de obtener capital para su proyecto. En España se hubiera planteado como un desarrollo local sin ánimo expansionista y la idea hubiera acabo siendo comprada para su desarrollo internacional. El creador de Uber ha encontrado apoyo financiero rápidamente para crear un elemento disruptivo en un sector muy tradicional y poco dado a los cambios. A partir de ahí, ya no es una start up, es una empresa con una dimensión distinta. Este salto es muy difícil darlo en España, que además tiene el handicap de que Europa tampoco es un mercado homogéneo. Si algo arranca en España, tiene el destino de ser comprado.

O.M. /J.M.G.: ¿La tecnología hace que las empresas sean más transparentes? ¿Qué papel juega la transparencia en este entorno?

E.D.: En el caso de España, vemos que las empresas tienen poca cultura de la transparencia. La sensación que tienes es que los entornos empresariales en realidad tienen miedo a la transparencia, cuando lo que debería hacerse es, a partir de un mínimo sentido común para no dar ventajas innecesarias a la competencia, conseguir que tu cliente entienda lo que haces, por qué lo haces y sobre todo qué es lo que estás haciendo con aquello que les has pedido, con su información.

Cada vez nos volvemos más sensibles en este sentido. La empresa que más sabe de nosotros, Google, es una organización en la que el común de los mortales confía porque resulta que por más información que sabemos 
que tiene, vemos que la utiliza bastante bien. La utiliza como mucho para acercarnos los anuncios que más nos interesan en un determinado momento. Esto puede ser bueno. Nos puede interesar. Si veo que me persigue, que me acosa, que me llama a la hora de cenar... entonces no me interesa. Si en este aspecto comparamos a Google con, por ejemplo, compañías de telefonía, vemos grandes diferencias. Google es mucho más respetuoso con tus datos.

Nos hace falta plantear las relaciones empresariales como relaciones humanas. Yo no puedo relacionarme razonablemente bien con alguien con el que cada vez que lo encuentro me dice "que grande soy...", "soy el mejor...", "soy el líder...". Pues estas empresas nos hablan así. Y cuando queremos solucionar un problema nos ponen por delante cuatro capas hasta llegar a una persona. Es absurdo. ¿Cómo quieren que confiemos en ellas?

O.M. / J.M.G.: Sobre la dimensión que está adquiriendo Google se incrementan ciertos temores ¿no existe el riesgo de que actúe como monopolio en muchas actividades? ¿No actúa en connivencia con las administraciones a la hora de obtener información sobre los ciudadanos? ¿Es transparente en sus declaraciones fiscales?

E.D.: Fiscalmente, Google hace lo mismo que cualquier otra compañía de su tamaño y dimensión: aprovechar la legislación vigente. Si no nos gusta lo que hace, no hay que atacar a Google, hay que cambiar la legislación. Lo contrario es una hipocresía: "sigue la ley estrictamente, optimiza su gasto fiscal... pero tengo que impedirlo". Las leyes tienen que ser para todos, para bien y para mal. Con respecto a si es o no un monopolio, no cabe duda de que, a medida que incrementa su poder de negociación y su escala, se convierte en un elefante en una cacharrería, que prácticamente ya no puede moverse sin romper algo. Las autoridades de defensa de la competencia deben estudiar esos movimientos y asegurarse de que restringen su capacidad de competir de manera predatoria o de apalancarse en su volumen para perjudicar a sus competidores, pero deben intentar no restringir su capacidad de innovación. Es, sin duda, un balance delicado.

O.M. / J.M.G.: ¿Cómo se está adaptando la universidad a estos cambios? ¿Los MOOC son un camino a seguir?

E.D.: Hay universidades en las que se enseña Contabilidad y llevan sus propias cuentas muy mal... Aquí puede ocurrir algo parecido. El problema de muchas universidades es la rigidez y la motivación. En Estados Unidos hay profesores para los que dar clase es lo menos importante. Y en España, hay una estructura rígida, con profesorado con plaza fija, que no facilita la adaptación porque no acepta cambios fácilmente. Los profesores deben ser evaluados con métricas de eficiencia como en cualquier organización, entre las que deben figurar de manera prominente la valoración de los estudiantes. En nuestro caso es así, y por lo tanto, estamos permanentemente pendientes de la mejora continua.

Por otro lado, los MOOC son aire fresco, pero no la solución final. Me niego a pensar que los son, porque no creo en la masificación absoluta. Nosotros en el IE trabajamos con una metodología de cursos online que va en dirección contraria: nuestros cursos online son incluso más caros que los presenciales, porque la profundidad de las discusiones es mayor. La metodología online no se basa en la memorización sino que exige ejercicios que no sean repetibles, intensificar el feedback, consultar y verificar fuentes... además la sensación de compañerismo, de compartir entre los estudiantes de los cursos online me fascina, porque llega a ser mayor que en grupos presenciales. Y es imposible trasladar esta eficiencia a los MOOC. No digo que vayan a fracasar, pueden funcionar con determinadas finalidades, pero no creo que representen el futuro. 


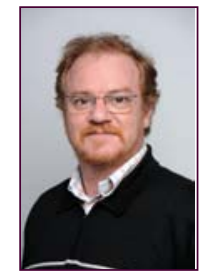

\section{Oriol Miralbell}

Doctor en Información y Sociedad del Conocimiento por la Universitat Oberta de Catalunya. Licenciado en Filología Alemana, máster en Gestión de la Información por la Universidad de Syracuse. Es profesor de los Estudios de Economía y Empresa de la UOC. Ha sido responsable de los servicios de información de la Dirección General de Turismo de la Generalitat de Cataluña. Es presidente del Capítulo español de la International Federation for IT and Travel \& Tourism (IFFIT), miembro del grupo de investigación GRATET (http://www.urv.cat/dgeo/gratet/) sobre Turismo y Geografía, miembro del Laboratorio del Nuevo Turismo (http: //turismo.blogs.uoc. edu / 3-recerca-id /). Centra su investigación en las TIC y el turismo, la gestión de los destinos y las redes sociales y el conocimiento.

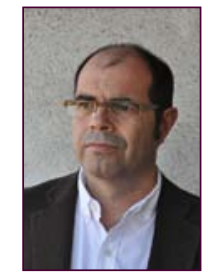

\section{Joan Miquel Gomis}

Director de Oikonomics. Doctor por el departamento de Economía y Organización de Empresas de la UB. Licenciado en Ciencias de la Información por la UAB. Director del programa (grado) de Turismo de los Estudios de Economía y Empresa de la UOC. Ha sido codirector académico (2006-2010) del máster sobre Gestión, política y estrategia de los destinos turísticos, organizado conjuntamente por la UOC y la Organización Mundial del Turismo (OMT). Sus áreas de especialización se sitúan en el ámbito de la dirección estratégica de la empresa turística, la aplicación de las TIC en la distribución y en el ámbito de la responsabilidad social. Investigador del grupo consolidado GRATET (http://www.urv.cat/dgeo/gratet/) y miembro del Laboratorio del Nuevo Turismo de la UOC.

Los textos publicados en esta revista están -si no se indica lo contrario- bajo una licencia Reconocimiento-Sin obras derivadas 3.0 España de Creative Commons. Puede copiarlos, distribuirlos y comunicarlos públicamente siempre que cite su autor y la revista y la institución que los publica (autoría, nombre de la revista, institución editora); no haga con ellos obras derivadas. La licencia completa se puede consultar en http://creativecommons.org/licenses/by-nd/3.0/es/deed.es.

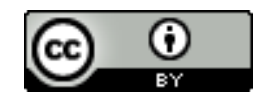

